QUARTERLY OF APPLIED MATHEMATICS

VOLUME LXVI, NUMBER 2

JUNE 2008, PAGES 359-377

S 0033-569X(08)01081-3

Article electronically published on February 29, 2008

\title{
STRONG DISCONTINUITIES FOR THE 2-D MEP HYDRODYNAMICAL MODEL OF CHARGE TRANSPORT IN SEMICONDUCTORS
}

BY

A. M. BLOKHIN

Institute of Mathematics, Novosibirsk 630090, Russia

\begin{abstract}
For the balance equations of charge transport in semiconductors based on the maximum entropy principle (see Anile and Romano (1999) and Romano (2000)), we derive and study the Rankine-Hugoniot jump conditions.
\end{abstract}

1. Introduction. In the hierarchy of macroscopic models of charge transport in semiconductors beyond the drift-diffusion equations [3, 4, 5] and the energy-transport models [6, 7, 8, one finds the hydrodynamical models that are obtained from an infinite set of moment equations of the Boltzmann Transport Equation by a suitable truncation procedure (see [9, 10] for a complete review on the mathematical modelling for semiconductors).

It is well known that moment systems require a closure assumption in order to yield a closed system of evolution equations. In [1, 2] the balance equations for density, momentum, energy, and energy flux of electrons have been considered for silicon and, by using the maximum entropy principle (MEP) for the closure, explicit constitutive relations for the stress tensor, the flux of energy flux tensor, and production terms have been obtained both in the parabolic and the Kane dispersion relation approximations for the energy conduction bands. The model fits into Extended Thermodynamics [10, 11, 12 ] and Levermore's theory of moments [13] and does not contain any fitting parameters. The validity of the constitutive equations has been checked in [14, 15] and the general mathematical properties have been studied in [16, where it has been shown that the evolution equations form a hyperbolic system in the physically relevant region of the field space. Applications of the model have been presented in [15, 16, for 1-D problems and in 17] for a 2-D simulation of a silicon MESFET (metal semiconductor field effect transistor). At last, the mathematical aspects of the model related to the stability of the equilibrium state for a 2-D silicon MESFET have been investigated in [18.

In the present paper, we continue the study of the system of balance equations proposed in [1, 2]. We are interested in piecewise smooth solutions of this system with smooth parts separated by a surface of strong discontinuity. Using a standard approach

Received November 11, 2006.

2000 Mathematics Subject Classification. Primary 76X05; Secondary 35B35, 35Q35, 82D37.

(C)2008 Brown University 
(see [19, 20, 21]), we obtain jump conditions for the balance equations under consideration.

The plan of the paper is the following. In Section 2 we write down the basic equations from [1, 2] and obtain the Rankine-Hugoniot jump conditions for them. In Section 3, we analyze these conditions for the particular case where the strong discontinuity is planar. The case of stationary planar discontinuity is discussed in Section 4.

Note also that various mathematical aspects of the model [1,2] related to the stability of the equilibrium state for a 1-D $n^{+}-n-n^{+}$silicon ballistic diode were studied in [22, 23, 24].

2. Basic equations and jump conditions. The mathematical model is given by the balance equations of density, momentum, energy, and energy flux of electrons [1, 2] closed by using the MEP. In the parabolic approximation of energy bands (see [16, 17]) it reads (in this paper we consider the 2 -D case)

$$
\begin{aligned}
& \frac{\partial n}{\partial t}+\operatorname{div}(n \mathbf{V})=0, \\
& \frac{\partial(n \mathbf{P})}{\partial t}+\nabla\left(\frac{2}{3} n W\right)+n e \mathbf{E}=n \mathbf{C}_{\mathbf{P}}, \\
& \frac{\partial(n W)}{\partial t}+\operatorname{div}(n \mathbf{S})+n e \cdot(\mathbf{E}, \mathbf{V})=n C_{W}, \\
& \frac{\partial(n \mathbf{S})}{\partial t}+\nabla\left(\frac{10}{9} n \frac{W^{2}}{m^{*}}\right)+\frac{5}{3} n e \frac{W}{m^{*}} \mathbf{E}=n \mathbf{C}_{\mathbf{W}},
\end{aligned}
$$

where $n$ is the electron density, $\mathbf{V}=\left(V^{(x)}, V^{(y)}\right)$ is the average electron velocity, $\mathbf{P}=m^{*} \mathbf{V}$ is the average momentum, $m^{*}$ is the effective electron mass (for silicon $m^{*}=0.32 m_{e}$ with $m_{e}$ being the mass of an electron in the vacuum; see Appendix), $W$ is the average electron energy, $\mathbf{S}=\left(S^{(x)}, S^{(y)}\right)$ is the energy flux, $e$ is the absolute value of the electron charge (see Appendix), $\mathbf{E}=\left(E^{(x)}, E^{(y)}\right)$ is the electric field, $\mathbf{C}_{\mathbf{P}}=\left(C_{P}^{(x)}, C_{P}^{(y)}\right)$ is the production of the crystal momentum balance equations, $C_{W}$ is the production of the energy balance equation, and $\mathbf{C}_{\mathbf{W}}=\left(C_{W}^{(x)}, C_{W}^{(y)}\right)$ is the production of the energy flux balance equations.

Since the electric field $\mathbf{E}$ is related to the electric potential $\Phi$ by

$$
\mathbf{E}=-\nabla \Phi=-\left(\Phi_{x}, \Phi_{y}\right),
$$

system (2.1 a $-2.1 \mathrm{~d}$ ) is coupled with the Poisson equation

$$
\epsilon \triangle \Phi=e(n-N) .
$$

Here $N=N_{D}-N_{A}$ is the doping density, $N_{D}$ and $N_{A}$ are the donor and acceptor densities respectively, and $\epsilon$ is the dielectric constant (see Appendix). 
Let us introduce the dimensionless variables

$$
\begin{gathered}
R=\frac{n}{N^{+}}, \quad \mathbf{u}=\left(u^{(x)}, u^{(y)}\right)=\frac{\mathbf{V}}{C_{0}}, \\
E=\frac{W}{m^{*} C_{0}^{2}}, \quad \mathbf{q}=\left(q^{(x)}, q^{(y)}\right)=\frac{\mathbf{S}}{m^{*} C_{0}^{3}}, \\
\varphi=\frac{\Phi e}{m^{*} C_{0}^{2}}, \quad \tau=\frac{C_{0} t}{L}, \quad \tilde{x}=\frac{x}{L}, \quad \tilde{y}=\frac{y}{L},
\end{gathered}
$$

where $R, E, \varphi, \mathbf{u}$, and $\mathbf{q}$ are new dependent variables, $\tau, \tilde{x}, \tilde{y}$ are new independent variables (in the following we will write $x, y$ instead of $\tilde{x}, \tilde{y}$ ), $N^{+}, L$ are characteristic density and length respectively, $C_{0}=\sqrt{\frac{K_{B} T_{0}}{m^{*}}}$ is a sort of sound speed, $T_{0}$ is the lattice temperature, and $K_{B}$ is the Boltzmann constant.

The values $T_{0}, K_{B}, L, N^{+}$are written in the Appendix. We also observe that the main results of this paper don't depend on the choice of the characteristic values $L$ and $N^{+}$.

The evolution equations in dimensionless form read

$$
\begin{aligned}
& R_{\tau}+\operatorname{div} \mathbf{J}=0, \\
& \mathbf{J}_{\tau}+\nabla\left(\frac{2}{3} R E\right)=R \mathbf{Q}+R \mathbf{S}_{\mathbf{P}}, \\
& (R E)_{\tau}+\operatorname{div} \mathbf{I}=(\mathbf{J}, \mathbf{Q})+R \widetilde{C}_{W}, \\
& \mathbf{I}_{\tau}+\nabla\left(\frac{10}{9} R E^{2}\right)=\frac{5}{3} R E \mathbf{Q}+R \mathbf{S}_{\mathbf{W}}, \\
& \triangle \varphi=\operatorname{div} \mathbf{Q}=\beta(R-\rho) .
\end{aligned}
$$

Here

$$
\begin{aligned}
& \mathbf{J}=R \mathbf{u}, \quad \mathbf{Q}=\nabla \varphi, \quad \mathbf{I}=R \mathbf{q}, \\
& \mathbf{S}_{\mathbf{P}}=\left(\widetilde{C}_{P}^{(x)}, \quad \widetilde{C}_{P}^{(y)}\right), \quad \mathbf{S}_{\mathbf{W}}=\left(\begin{array}{l}
\widetilde{C}_{W}^{(x)}, \quad \widetilde{C}_{W}^{(y)}
\end{array}\right), \\
& \widetilde{C}_{P}^{(x),(y)}=C_{P}^{(x),(y)} \frac{L}{m^{*} C_{0}^{2}}, \quad \widetilde{C}_{W}=C_{W} \frac{L}{m^{*} C_{0}^{3}}, \quad \widetilde{C}_{W}^{(x),(y)}=C_{W}^{(x),(y)} \frac{L}{m^{*} C_{0}^{4}}, \\
& \rho=\rho(x, y)=\frac{N}{N^{+}}, \quad \beta=\frac{e^{2} L^{2} N^{+}}{\epsilon m^{*} C_{0}^{2}} .
\end{aligned}
$$

The expressions for the production terms $\mathbf{C}_{\mathbf{P}}, C_{W}, \mathbf{C}_{\mathbf{W}}$ have been obtained in [2] (for more details the interested reader is referred to [16, 17]). In dimensionless form we have the following expressions for the production terms (see also [22, 23]):

$$
\left.\begin{array}{l}
\left(\begin{array}{l}
\widetilde{C}_{P}^{(x),(y)} \\
\widetilde{C}_{W}^{(x),(y)}
\end{array}\right)=\left(\begin{array}{ll}
c_{11} & c_{12} \\
c_{21} & c_{22}
\end{array}\right)\left(\begin{array}{l}
u^{(x),(y)} \\
q^{(x),(y)}
\end{array}\right), \\
R \widetilde{C}_{W}=c P, \quad P=R\left(\frac{2}{3} E-1\right) .
\end{array}\right\}
$$

The explicit expressions for the coefficients $c_{11}, c_{12}, c_{21}, c_{22}$, and $c$ are reported in [22, 23] (see also the Appendix). Note that these coefficients are smooth functions of $E$. 
Before getting the jump conditions for system (2.3 a $-(2.3 \mathrm{~d})$, (2.4), we give equivalent forms for some equations of this system (they are equivalent on smooth solutions). Indeed, in view of the Poisson equation (2.4), one has

$$
R \mathbf{Q}=\rho \mathbf{Q}+(R-\rho) \mathbf{Q}=\rho \mathbf{Q}+\varepsilon \operatorname{div} \mathbf{Q} \mathbf{Q},
$$

where $\varepsilon=\frac{1}{\beta}$ ( $\sqrt{\varepsilon}$ is the so-called Debye length reduced to a dimensionless form; see [19]). Since

$$
\operatorname{div} \mathbf{Q} \mathbf{Q}=-\nabla\left(\frac{|\mathbf{Q}|^{2}}{2}\right)+\operatorname{div}(\mathbf{Q} \otimes \mathbf{Q}),
$$

then, by virtue of (2.6),$(2.7)$, equation (2.3 b) takes the form (see also formulae (2.5)):

$$
\mathbf{J}_{\tau}-\varepsilon \operatorname{div}(\mathbf{Q} \otimes \mathbf{Q})+\nabla\left(\frac{2}{3} R E+\varepsilon \frac{|\mathbf{Q}|^{2}}{2}\right)=\rho \mathbf{Q}+c_{11} \mathbf{J}+c_{12} \mathbf{I} .
$$

Here $\operatorname{div}(\mathbf{Q} \otimes \mathbf{Q})=\left(\operatorname{div}\left(\varphi_{x} \mathbf{Q}\right), \operatorname{div}\left(\varphi_{y} \mathbf{Q}\right)\right)$.

Then, differentiating the Poisson equation (2.4) with respect to $\tau$ and taking into account relation (2.3 a), we can introduce the vector $\mathbf{J}^{*}$ that is connected with $\mathbf{Q}$ and $\mathbf{J}$ by the formula

$$
\mathbf{J}^{*}=\mathbf{J}+\varepsilon \mathbf{Q}_{\tau} .
$$

Moreover, the vector $\mathbf{J}^{*}$ satisfies the relations

$$
\left.\begin{array}{l}
\operatorname{div} \mathbf{J}^{*}=0 \\
\operatorname{rot}_{z}\left(\mathbf{J}^{*}-\mathbf{J}\right)=0,
\end{array}\right\}
$$

where $\operatorname{rot}_{z} \mathbf{A}=A_{x}^{(y)}-A_{y}^{(x)}, \mathbf{A}=\left(A^{(x)}, A^{(y)}\right)$. The last relation is fulfilled because $\operatorname{rot}_{z} \mathbf{Q}=0$.

Hence, knowing $\mathbf{J}$ and $\mathbf{J}^{*}$, we find the vector $\mathbf{Q}$ from (2.8) (in this case the Poisson equation (2.4) is treated as a condition on the initial data). Taking into account (2.8), equation $(2.3 \mathrm{c})$ is rewritten as

$$
\left(R E+\varepsilon \frac{|\mathbf{Q}|^{2}}{2}\right)_{\tau}+\operatorname{div} \mathbf{I}=\left(\mathbf{J}^{*}, \mathbf{Q}\right)+c P .
$$

At last, instead of the vector equation $2.3 \mathrm{~d}$ ) we can use another one if necessary. Namely, multiplying equation (2.3 b) by $\frac{5}{2} \varphi$ and subtracting it from (2.3 d), we get

$$
\left.\boldsymbol{\Theta}_{\tau}+\nabla\left(\frac{10}{9} R E^{2}-\frac{5}{3} R E \varphi\right)=R \mathbf{S}_{\mathbf{W}}-\frac{5}{2} \varphi\left(R \mathbf{S}_{\mathbf{P}}+R \mathbf{Q}\right)-\frac{5}{2} \varphi_{\tau} \mathbf{J}, \boldsymbol{\Theta}=\mathbf{I}-\frac{5}{2} \mathbf{J} \varphi .2 .3 \mathrm{~d}\right)
$$

We are now interested in piecewise smooth solutions to system (2.3 a d , $2.3 \mathrm{~b}$ ), (2.3 cll), (2.3 d), (2.4), a solution in which smooth parts are separated by a surface of strong discontinuity

$$
\tilde{f}(\tau, x, y)=f(\tau, y)-x=0 .
$$

As is known (see [19, 20, 21]), jump conditions, usually called Rankine-Hugoniot conditions, should be satisfied on the surface of strong discontinuity (2.10). Let us first formulate them for an abstract system of balance laws

$$
\frac{\partial \mathcal{P}_{i}^{(0)}}{\partial \tau}+\operatorname{div} \mathcal{P}_{i}=\mathcal{F}_{i}, i=\overline{1, l},
$$


where $\mathcal{P}_{i}=\left(\mathcal{P}_{i}^{(x)}, \mathcal{P}_{i}^{(y)}\right), \mathcal{P}_{i}^{(\alpha)}=\mathcal{P}_{i}^{(\alpha)}(\mathbf{U}), \alpha=0, x, y, \mathcal{F}_{i}=\mathcal{F}_{i}(\tau, x, y, \mathbf{U})$, and $\mathbf{U}=$ $\left(u_{1}, \ldots, u_{l}\right)$ is the vector of unknowns. Let us suppose that

$$
\mathcal{F}_{i}(\tau, x, y, \mathbf{U}(\tau, x, y))=0, i=\overline{1, l},
$$

for smooth parts of the considered piecewise smooth solutions of system (2.11) (it corresponds to the case of piecewise constants equilibria studied in Section 3). Then, following to [20, the jump conditions for the system of balance laws (2.11) have the form

$$
\left[\mathcal{P}_{i N}\right]=D_{N}\left[\mathcal{P}_{i}^{(0)}\right], i=\overline{1, l} .
$$

Here $\mathcal{P}_{i N}=\left(\mathcal{P}_{i}, \mathbf{N}\right), \mathbf{N}=\frac{\left(-1, f_{y}\right)}{|\nabla f|}$ is the unit normal to the discontinuity front (2.10), $D_{N}=-\frac{f_{\tau}}{|\nabla \tilde{f}|}$ is the discontinuity speed in the normal direction, $|\nabla \tilde{f}|^{2}=1+f_{y}^{2}$, and the notation $[F]$ is usual, i.e., $[F]=F-F_{\infty}$, where $F$ and $F_{\infty}$ are respectively the limit values of $F$ from the right $(\tilde{f} \rightarrow-0)$ and from the left $(\tilde{f} \rightarrow+0)$ of the discontinuity (2.10).

Specifying the functions $\mathcal{P}_{i}^{(\alpha)}, \alpha=0, x, y, i=\overline{1, l}$, for system (2.3 a a, $2.3 \mathrm{~b}$, $2.3 \mathrm{cll}$, $(2.3 \mathrm{~d}),(2.4)$, we get the jump conditions

$$
\begin{aligned}
& {\left[R\left(u_{N}-D_{N}\right)\right]=\frac{\partial \omega}{\partial \tau}+\operatorname{div}_{\sigma} \mathbf{i},} \\
& \frac{2}{3}[R E]=\left[R u_{N}\right] D_{N}+\varepsilon\left[Q_{N}^{2}-\frac{|\mathbf{Q}|^{2}}{2}\right], \\
& {\left[R u_{\sigma}\right] D_{N}+\varepsilon\left[Q_{\sigma} Q_{N}\right]=0,} \\
& {\left[R q_{N}\right]=\left[R E+\varepsilon \frac{|\mathbf{Q}|^{2}}{2}\right] D_{N}+\operatorname{div}_{\sigma} \mathbf{p},} \\
& {\left[R q_{\sigma}\right] D_{N}=0,} \\
& \frac{10}{9}\left[R E^{2}\right]=\left[R q_{N}\right] D_{N}, \\
& \varepsilon\left[Q_{N}\right]=-\omega, \\
& {\left[Q_{\sigma}\right]=0}
\end{aligned}
$$

that should be satisfied on the discontinuity front (2.10), where $u_{N}=(\mathbf{u}, \mathbf{N}), Q_{N}=$ $(\mathbf{Q}, \mathbf{N}), q_{N}=(\mathbf{q}, \mathbf{N}), u_{\sigma}=(\mathbf{u}, \boldsymbol{\sigma}), Q_{\sigma}=(\mathbf{Q}, \boldsymbol{\sigma}), q_{\sigma}=(\mathbf{q}, \boldsymbol{\sigma}), \boldsymbol{\sigma}=\left(f_{y}, 1\right)$, and $(\boldsymbol{\sigma}, \mathbf{N})=0$. If we take (2.3 dill instead of (2.3 d), conditions (2.14 el) and (2.14 fl) should be changed to

$$
\begin{gathered}
{\left[\Theta_{\sigma}\right] D_{N}=0, \Theta_{\sigma}=(\boldsymbol{\Theta}, \sigma),} \\
\frac{10}{9}\left[R E^{2}\right]=\left[Q_{N}\right] D_{N}+\frac{5}{3}[R E \varphi], \Theta_{N}=(\boldsymbol{\Theta}, \mathbf{N}) .
\end{gathered}
$$

Moreover, one more jump condition is the condition of continuity of the electric potential $\varphi$ :

$$
[\varphi]=0 .
$$

Taking into account a specific character of strong discontinuities under the presence of an electric field (see [19, 21]), in the jump conditions (2.14 a $-2.14 \mathrm{~h}$ ) we introduce 
some values corresponding to the possible existence of a surface charge $\omega=\omega(\tau, y)$, a surface current $\mathbf{i}=\mathbf{i}(\tau, y)$, and a surface energy flux $\mathbf{p}=\mathbf{p}(\tau, y)$ (2.10). The approach for the introduction of such values is detailed in [19, 21. Moreover, $\operatorname{div}_{\sigma}$ appearing in (2.14 a $),(2.14 \mathrm{~d}$ ) is the so-called surface divergence (see [21]).

Following [21, we specify the values $\operatorname{div}_{\sigma} \mathbf{i}, \operatorname{div}_{\sigma} \mathbf{p}$. The squared arc length for two infinitely near points lying on the discontinuity front (2.10) is as follows:

$$
(d s)^{2}=|\nabla \tilde{f}|^{2}(d y)^{2}=g_{11}\left(d \xi^{1}\right)^{2}, g_{11}=|\nabla \tilde{f}|^{2}, \xi^{1}=y .
$$

Consequently, identifying the vector $\boldsymbol{\sigma}$ with the covariant vector $\boldsymbol{\epsilon}_{1}$ of the basis introduced on surface (2.10), one has

$$
\left.\begin{array}{l}
\operatorname{div}_{\sigma} \mathbf{i}=\frac{1}{\sqrt{g_{11}}} \frac{\partial\left(i^{*}(\tau, y) \sqrt{g_{11}}\right)}{\partial y}, \mathbf{i}=i^{*} \boldsymbol{\epsilon}_{1}, \\
\operatorname{div}_{\sigma} \mathbf{p}=\frac{1}{\sqrt{g_{11}}} \frac{\partial\left(p^{*}(\tau, y) \sqrt{g_{11}}\right)}{\partial y}, \mathbf{p}=p^{*} \boldsymbol{\epsilon}_{1} .
\end{array}\right\}
$$

Here $i^{*}, p^{*}$ are contravariant components of the vectors $\mathbf{i}, \mathbf{p}$.

We note that the procedure of introduction of surface charge, surface current, and surface energy flux in the jump conditions (2.14 a $-2.14 \mathrm{~h}$ ) still looks rather formal. However, in view of conditions (2.12), in the next section we show that the surface electric current and the surface energy flux appearing on the discontinuity front are finally determined by the surface charge.

3. Study of the jump conditions for a planar discontinuity. Let us consider a planar discontinuity with the equation

$$
\hat{d} \tau-x=0,
$$

where $\hat{d}$ is a constant (the discontinuity speed; see Fig. 1). Consider also a piecewise constant solution,

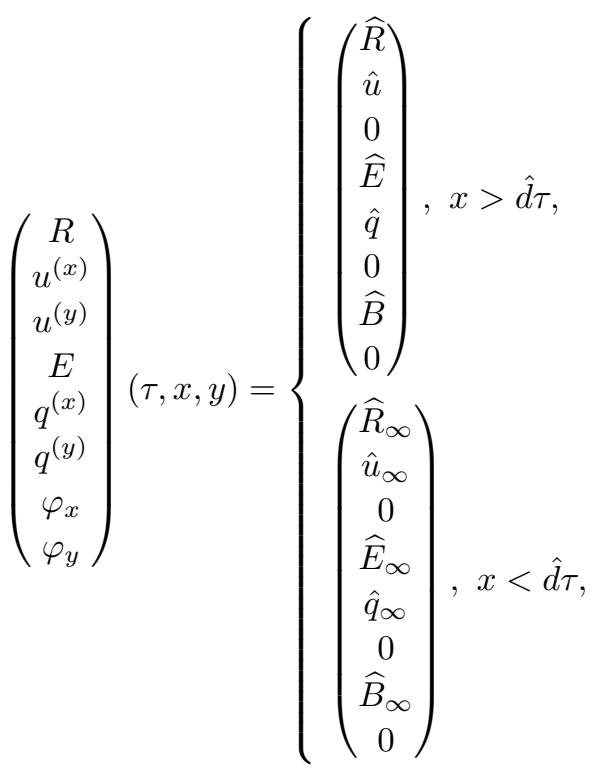




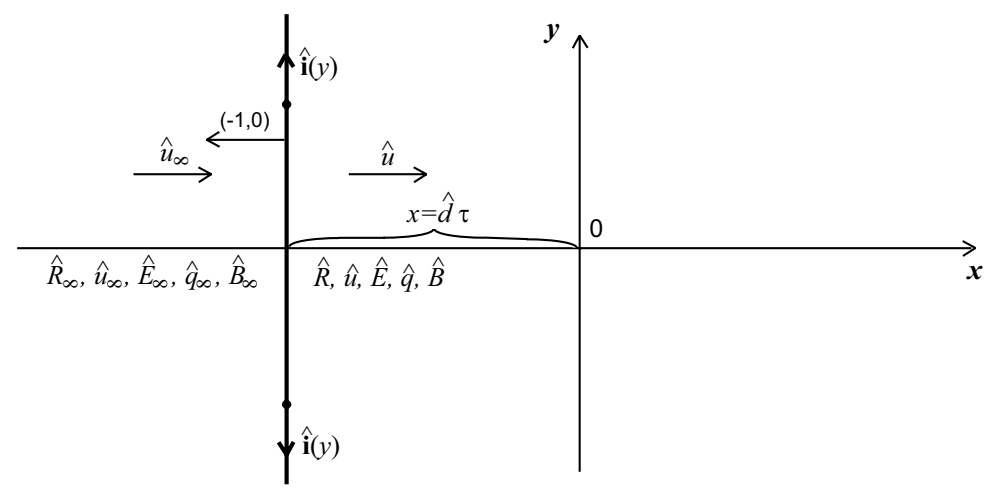

FIG. 1. Planar discontinuity

to system (2.3 a $-(2.3 \mathrm{~d}),(2.4)$. If $\hat{d}<0$, then the discontinuity moves from right to left (see Fig. 1).

The constants $\widehat{R}, \hat{u}, \widehat{E}, \hat{q}, \widehat{B}, \widehat{R}_{\infty}, \hat{u}_{\infty}, \widehat{E}_{\infty}, \hat{q}_{\infty}$, and $\widehat{B}_{\infty}$ satisfy system (2.3 a $-(2.3 \mathrm{~d})$, (2.4); i.e., they must meet conditions (2.12):

$$
\begin{aligned}
& \left.\begin{array}{l}
\left(\begin{array}{ll}
c_{11} & c_{12} \\
c_{21} & c_{22}
\end{array}\right)(\widehat{E})\left(\begin{array}{l}
\hat{u} \\
\hat{q}
\end{array}\right)+\widehat{B}\left(\begin{array}{c}
1 \\
\frac{5}{3} \widehat{E}
\end{array}\right)=0, \\
c(\widehat{E})\left(\frac{2}{3} \widehat{E}-1\right)+\widehat{B} \hat{u}=0,
\end{array}\right\} \\
& \left(\begin{array}{ll}
c_{11} & c_{12} \\
c_{21} & c_{22}
\end{array}\right)\left(\widehat{E}_{\infty}\right)\left(\begin{array}{l}
\hat{u}_{\infty} \\
\hat{q}_{\infty}
\end{array}\right)+\widehat{B}_{\infty}\left(\begin{array}{c}
1 \\
\frac{5}{3} \widehat{E}_{\infty}
\end{array}\right)=0, \\
& c\left(\widehat{E}_{\infty}\right)\left(\frac{2}{3} \widehat{E}_{\infty}-1\right)+\widehat{B}_{\infty} \hat{u}_{\infty}=0 .
\end{aligned}
$$

We suppose that the potential $\varphi$ is continuous on the discontinuity (3.1), and we seek it in the form

$$
\varphi(\tau, x, y)=\left\{\begin{array}{l}
\widehat{B}_{\infty} x, x<\hat{d} \tau \\
\widehat{B} x-[\widehat{B}] \hat{d} \tau, x>\hat{d} \tau
\end{array}\right.
$$

where $[\widehat{B}]=\widehat{B}-\widehat{B}_{\infty}$. Finally, for the fulfillment of equation (2.4) we suppose that the doping density $\rho(x, y)$ is as follows:

$$
\rho(x, y)=\left\{\begin{array}{l}
\widehat{R}, x>\hat{d} \tau \\
\widehat{R}_{\infty}, x<\hat{d} \tau
\end{array}\right.
$$


The constants $\widehat{R}, \hat{u}, \widehat{E}, \hat{q}, \widehat{B}, \widehat{R}_{\infty}, \hat{u}_{\infty}, \widehat{E}_{\infty}, \hat{q}_{\infty}$, and $\widehat{B}_{\infty}$ should satisfy not only relations (3.3 a $),(3.3 \mathrm{~b})$ but also the jump conditions (2.14 a $)-(2.14 \mathrm{~h})$ that can be transformed to the form

$$
\begin{aligned}
& \bar{R} \hat{u}-\hat{u}_{\infty}=\widehat{\Delta} \hat{d}+\hat{j}, \\
& \frac{2}{3}\left(\bar{R} \widehat{E}-\widehat{E}_{\infty}\right)=\widehat{\Delta} \hat{d}^{2}+\hat{\lambda}\left[\widehat{B}^{2}\right]+\hat{j} \hat{d}, \\
& \bar{R} \hat{q}-\hat{q}_{\infty}=\left(\bar{R} \widehat{E}-\widehat{E}_{\infty}\right) \hat{d}+\hat{\lambda}\left[\widehat{B}^{2}\right] \hat{d}+\hat{g}, \\
& \frac{10}{9}\left(\bar{R} \widehat{E}^{2}-\widehat{E}_{\infty}^{2}\right)=\left(\bar{R} \widehat{E}-\widehat{E}_{\infty}\right) \hat{d}^{2}+\hat{\lambda}\left[\widehat{B}^{2}\right] \hat{d}^{2}+\hat{g} \hat{d}, \\
& \varepsilon[\widehat{B}]=\hat{\omega} .
\end{aligned}
$$

$$
\begin{array}{ll}
\text { Here } & \bar{R}=\frac{\widehat{R}}{\widehat{R}_{\infty}}, \quad \widehat{\Delta}=\bar{R}-1, \quad \hat{\lambda}=\frac{\varepsilon}{2 \widehat{R}_{\infty}}, \\
\hat{j}=-\frac{1}{\widehat{R}_{\infty}} \frac{d \hat{i}^{*}(y)}{d y}=\text { const, } \\
\hat{\mathbf{i}}(y)=-\hat{j} y \widehat{R}_{\infty} \hat{\boldsymbol{\epsilon}}_{1}, \quad \hat{\boldsymbol{\epsilon}}_{1}=(0,1), \\
\hat{\mathbf{i}}(y) \text { is the surface current (see Fig. } 1), \\
\hat{g}=-\frac{1}{\widehat{R}_{\infty}} \frac{d \hat{p}^{*}(y)}{d y}=\text { const, } \\
\hat{\mathbf{p}}(y)=-\hat{g} y \widehat{R}_{\infty} \hat{\boldsymbol{\epsilon}}_{1}, \\
\hat{\mathbf{p}}(y) \text { is the surface energy flux, } \\
\hat{\omega}(=\text { const) is the surface charge. }
\end{array}
$$

It is convenient to rewrite relations $(3.3 \mathrm{a}), 3.3 \mathrm{~b}$ ) in the form

$$
\left.\begin{array}{l}
\left(\begin{array}{l}
\hat{u} \\
\hat{q}
\end{array}\right)=-\widehat{B}\left(\begin{array}{ll}
c_{11} & c_{12} \\
c_{21} & c_{22}
\end{array}\right)^{-1}\left(\begin{array}{c}
1 \\
\frac{5}{3} \widehat{E}
\end{array}\right)=\left(\begin{array}{l}
\widehat{B} \widehat{F} \\
\widehat{B} \widehat{G}
\end{array}\right)=\left(\begin{array}{c}
\widehat{B} \widehat{F} \\
\hat{u} \widehat{\Lambda}
\end{array}\right), \\
\left(\begin{array}{l}
\hat{u}_{\infty} \\
\hat{q}_{\infty}
\end{array}\right)=\left(\begin{array}{l}
\widehat{B}_{\infty} \widehat{F}_{\infty} \\
\widehat{B}_{\infty} \widehat{G}_{\infty}
\end{array}\right)=\left(\begin{array}{l}
\widehat{B}_{\infty} \widehat{F}_{\infty} \\
\hat{u}_{\infty} \widehat{\Lambda}_{\infty}
\end{array}\right), \\
\hat{u}^{2}=\widehat{U}, \quad \hat{u}_{\infty}^{2}=\widehat{U}_{\infty},
\end{array}\right\}
$$

where $\widehat{F}=F(\widehat{E}), \widehat{F}_{\infty}=F\left(\widehat{E}_{\infty}\right)$ and so on,

$$
\begin{aligned}
& F(E)=-\frac{c_{22}(E)-\frac{5}{3} E c_{12}(E)}{\operatorname{det}}, \quad G(E)=\frac{c_{21}(E)-\frac{5}{3} E c_{11}(E)}{\operatorname{det}}, \\
& \operatorname{det}=c_{11}(E) c_{22}(E)-c_{12}(E) c_{21}(E), \\
& \Lambda(E)=\frac{G(E)}{F(E)}, \quad U(E)=-c(E) F(E)\left(\frac{2}{3} E-1\right) .
\end{aligned}
$$


The graphs of the functions $c(E), F(E), G(E), \Lambda(E)$, and $U(E)$ for $E \geq \frac{3}{2}$ are presented in Fig. 2 (see the Appendix).

Let us now analyze conditions (3.6a)-(3.6e), (3.7). For simplicity we limit ourselves to the case of small-amplitude discontinuities. We will assume that the value $\hat{d}$ is small enough and the following expansions are true:

$$
\left.\begin{array}{l}
\bar{R}=1+\bar{R}_{2} \hat{d}^{2}+O\left(\hat{d}^{4}\right), \\
\hat{u}=\hat{u}^{(0)} \hat{d}+O\left(\hat{d}^{3}\right), \\
\hat{u}_{\infty}=\hat{u}_{\infty}^{(0)} \hat{d}+O\left(\hat{d}^{3}\right), \\
\widehat{E}=\frac{3}{2}+\hat{e} \hat{d}^{2}+O\left(\hat{d}^{4}\right), \\
\widehat{E}_{\infty}=\frac{3}{2}+\hat{e}_{\infty} \hat{d}^{2}+O\left(\hat{d}^{4}\right), \\
\hat{j}=\hat{j}_{0} \hat{d}+O\left(\hat{d}^{3}\right), \\
\hat{g}=\hat{g}_{0} \hat{d}+O\left(\hat{d}^{3}\right), \\
\hat{\omega}=\hat{\omega}_{0} \hat{d}+O\left(\hat{d}^{3}\right) .
\end{array}\right\}
$$

Substituting expansions (3.8) into relations (3.6a)-(3.6e), (3.7), we obtain step by step that

$$
\begin{aligned}
& \hat{u}^{(0)}-\hat{u}_{\infty}^{(0)}=\hat{j}_{0}, \\
& \hat{e}-\hat{e}_{\infty}=-\frac{3}{2} \bar{R}_{2}+\frac{3}{2} \hat{j}_{0}\left\{1+\frac{\hat{\lambda}}{F^{2}\left(\frac{3}{2}\right)}\left(\hat{u}^{(0)}+\hat{u}_{\infty}^{(0)}\right)\right\}, \\
& \hat{g}_{0}=\Lambda\left(\frac{3}{2}\right) \hat{j}_{0}, \\
& \hat{e}-\hat{e}_{\infty}=\frac{3}{10} \Lambda\left(\frac{3}{2}\right) \hat{j}_{0}-\frac{3}{4} \bar{R}_{2}, \\
& \varepsilon \frac{\hat{j}_{0}}{F\left(\frac{3}{2}\right)}=\hat{\omega}_{0}, \\
& \hat{e}=\frac{3\left(\hat{u}^{(0)}\right)^{2}}{2\left|c\left(\frac{3}{2}\right)\right| F\left(\frac{3}{2}\right)}, \quad \hat{e}_{\infty}=\frac{3\left(\hat{u}_{\infty}^{(0)}\right)^{2}}{2\left|c\left(\frac{3}{2}\right)\right| F\left(\frac{3}{2}\right)} .
\end{aligned}
$$

It follows from (3.9 c), (3.9 e that the surface energy flux and the surface charge are determined by the surface current (up to terms of order $O\left(\hat{d}^{3}\right)$ ).

Analyzing relations $(3.9 \mathrm{~b}),(3.9 \mathrm{~d}),(3.9 \mathrm{f})$, we deduce the solvability conditions

$$
\begin{aligned}
& \bar{R}_{2}=2 \hat{j}_{0}\left\{1-\frac{1}{5} \Lambda\left(\frac{3}{2}\right)-\frac{\hat{\lambda}}{F^{2}\left(\frac{3}{2}\right)} \hat{\mu}\right\}, \\
& \hat{u}^{(0)}+\hat{u}_{\infty}^{(0)}=-\hat{\mu} .
\end{aligned}
$$

Here

$$
\hat{\mu}=\frac{1-\frac{2}{5} \Lambda\left(\frac{3}{2}\right)}{F\left(\frac{3}{2}\right)+\hat{\lambda}\left|c\left(\frac{3}{2}\right)\right|}\left|c\left(\frac{3}{2}\right)\right| F^{2}\left(\frac{3}{2}\right)>0 \text { (see the Appendix). }
$$


Consequently, it follows from (3.9 a $)$, (3.10) that

$$
\left.\begin{array}{l}
\hat{u}^{(0)}=\frac{1}{2} \hat{j}_{0}-\frac{1}{2} \hat{\mu}, \\
\hat{u}_{\infty}^{(0)}=-\frac{1}{2} \hat{j}_{0}-\frac{1}{2} \hat{\mu}, \\
\bar{R}_{2}=2 \hat{j}_{0} \frac{\left(1-\frac{1}{5} \Lambda\left(\frac{3}{2}\right)\right) F\left(\frac{3}{2}\right)+\frac{1}{5} \hat{\lambda} \Lambda\left(\frac{3}{2}\right)\left|c\left(\frac{3}{2}\right)\right|}{F\left(\frac{3}{2}\right)+\hat{\lambda}\left|c\left(\frac{3}{2}\right)\right|}=2 \hat{j}_{0} \hat{\nu} .
\end{array}\right\}
$$

Let $\hat{d}<0, \hat{j}_{0}>0$ (see Fig. 1). For $\hat{j}_{0}<\hat{\mu}$ one has that $\hat{u}^{(0)}<0, \hat{u}_{\infty}^{(0)}<0$, and $\left|\hat{u}_{\infty}^{(0)}\right|>\left|\hat{u}^{(0)}\right|$. Then $\hat{u}>0, \hat{u}_{\infty}>0$, and $\hat{u}_{\infty}>\hat{u}$. Hence, there is an electron flow through the discontinuity, and

a) $\hat{e}_{\infty}>\hat{e}$ and $\widehat{E}_{\infty}>\widehat{E}$,

b) $\bar{R}_{2}>0$ (see the Appendix) and $\bar{R}>1, \widehat{R}>\widehat{R}_{\infty}$.

For $\hat{j}_{0}=\hat{\mu}$ the electron flow appears to be choking, i.e., $\hat{u}^{(0)}=0$ and $\hat{u}=0$ up to terms of order $O\left(\hat{d}^{3}\right)$. At last, electrons move in reverse direction while $\hat{j}_{0}$ increases $\left(\hat{j}_{0}>\hat{\mu}\right)$.

Summarizing arguments stated above, we observe that the consideration of strong discontinuity with a fixed constant surface charge generates a need for the introduction of surface current, which provides the carry-over of "superfluous" electrons from the discontinuity surface (see Fig. 1). Note also that we have determined only the first expansion terms in (3.8). Subsequent expansion terms could be easily found as well.

4. Stationary planar discontinuity. Analysis in Section 3 shows a principal possibility of existence of strong discontinuities for the 2-D MEP hydrodynamical model of charge transport in semiconductors. However, the case of moving doping profile considered in Section 3 seems to be not quite realistic (in spite of the fact that the shock velocity $\hat{d}$ is a small parameter). Therefore, in this section we study the case of stationary planar discontinuity.

Let us consider a planar discontinuity with the equation

$$
x=0,
$$

i.e., we suppose that $\hat{d}=0$ (see Section 3).

For the conservation laws (2.3 a), (2.3 bl), (2.3 cld), (2.3 dld, (2.4), we consider their piecewise constant solution (3.2) that should satisfy conditions (2.12):

$$
\begin{aligned}
& \left(\begin{array}{ll}
c_{11} & c_{12} \\
c_{21} & c_{22}
\end{array}\right)(\widehat{E})\left(\begin{array}{l}
\hat{u} \\
\hat{q}
\end{array}\right)+\widehat{B}\left(\begin{array}{l}
1 \\
0
\end{array}\right)=0, \\
& \left.c(\widehat{E})\left(\frac{2}{3} \widehat{E}-1\right)+\widehat{B} \hat{u}=0, \quad\right\} \\
& \left(\begin{array}{ll}
c_{11} & c_{12} \\
c_{21} & c_{22}
\end{array}\right)\left(\widehat{E}_{\infty}\right)\left(\begin{array}{l}
\hat{u}_{\infty} \\
\hat{q}_{\infty}
\end{array}\right)+\widehat{B}_{\infty}\left(\begin{array}{l}
1 \\
0
\end{array}\right)=0 \\
& \left.c\left(\widehat{E}_{\infty}\right)\left(\frac{2}{3} \widehat{E}_{\infty}-1\right)+\widehat{B}_{\infty} \hat{u}_{\infty}=0 . \quad\right\}
\end{aligned}
$$


We suppose that the potential $\varphi$ is continuous on the discontinuity $x=0$ (see condition (2.14 id), and seek it in the form

$$
\varphi(\tau, x, y)=\left\{\begin{array}{l}
\widehat{B}_{\infty} x+\widehat{A}, x<0, \\
\widehat{B} x+\widehat{A}, x>0,
\end{array}\right.
$$

where $\widehat{A}$ is a constant that will be determined below. Finally, for the fulfillment of equation (2.4), we suppose that the doping density $\rho(x, y)$ is as follows:

$$
\rho(x, y)=\left\{\begin{array}{l}
\widehat{R}, x>0, \\
\widehat{R}_{\infty}, x<0 .
\end{array}\right.
$$

The constants $\widehat{R}, \hat{u}, \widehat{E}, \hat{q}, \widehat{B}, \widehat{R}_{\infty}, \hat{u}_{\infty}, \widehat{E}_{\infty}, \hat{q}_{\infty}$, and $\widehat{B}_{\infty}$ (see (3.2)) should satisfy not only relations (4.1 a ), (4.1 b) but also the jump conditions (2.14 a $-(2.14 \mathrm{~d}$ ), (2.14 ell), (2.14 fll, $2.14 \mathrm{~g}),(2.14 \mathrm{~h})$ that can be transformed to the form

$$
\begin{aligned}
& \bar{R} \hat{u}-\hat{u}_{\infty}=\hat{j}, \\
& \frac{2}{3}\left(\bar{R} \widehat{E}-\widehat{E}_{\infty}\right)=\hat{\lambda}\left[\widehat{B}^{2}\right], \\
& \bar{R} \hat{q}-\hat{q}_{\infty}=\hat{g}, \\
& \frac{10}{9}\left(\bar{R} \widehat{E}^{2}-\widehat{E}_{\infty}^{2}\right)=\frac{5}{2} \widehat{A} \hat{\lambda}\left[\widehat{B}^{2}\right], \\
& \varepsilon[\widehat{B}]=\hat{\omega} .
\end{aligned}
$$

The values $\bar{R}, \hat{j}, \hat{\lambda}, \hat{g}, \hat{\omega}$ are described in Section 3 .

It is convenient to rewrite relations (4.1 a d $4.1 \mathrm{~b}$ ) in the form

$$
\left.\begin{array}{c}
\left(\begin{array}{l}
\hat{u} \\
\hat{q}
\end{array}\right)=\left(\begin{array}{l}
\widehat{B} \widehat{F} \\
\hat{u} \widehat{\Lambda}
\end{array}\right), \\
\left(\begin{array}{l}
\hat{u}_{\infty} \\
\hat{q}_{\infty}
\end{array}\right)=\left(\begin{array}{l}
\widehat{B}_{\infty} \widehat{F}_{\infty} \\
\hat{u}_{\infty} \widehat{\Lambda}_{\infty}
\end{array}\right), \\
\hat{u}^{2}=\widehat{U}, \quad \hat{u}_{\infty}^{2}=\widehat{U}_{\infty},
\end{array}\right\}
$$

where $\widehat{F}=F(\widehat{E}), \widehat{F}_{\infty}=F\left(\widehat{E}_{\infty}\right)$ and so on,

$$
\begin{aligned}
& F(E)=-\frac{c_{22}(E)}{\operatorname{det}}, \quad G(E)=\frac{c_{21}(E)}{\operatorname{det}}, \\
& \operatorname{det}=c_{11}(E) c_{22}(E)-c_{12}(E) c_{21}(E), \\
& \Lambda(E)=\frac{G(E)}{F(E)}=-\frac{c_{21}(E)}{c_{22}(E)}, \quad U(E)=-c(E) F(E)\left(\frac{2}{3} E-1\right) .
\end{aligned}
$$

The graphs of the functions $F(E), G(E), \Lambda(E)$, and $U(E)$ for $E \geq \frac{3}{2}$ are presented in Fig. 3 (see the Appendix).

Let us now analyze conditions 4.4 a - 4.4 e (4.5). As in Section 3 for simplicity we limit ourselves to the case of small-amplitude discontinuities. We will assume that the 
value $\hat{\Delta}$ is small (the value $\hat{\Delta}$ characterizes the strength of strong discontinuity) and the following expansions are true:

$$
\begin{aligned}
& \bar{R}=1+\bar{R}_{2} \hat{\Delta}^{2}+O\left(\hat{\Delta}^{4}\right), \\
& \hat{u}=\hat{u}^{(0)} \hat{\Delta}+O\left(\hat{\Delta}^{3}\right), \\
& \hat{u}_{\infty}=\hat{u}_{\infty}^{(0)} \hat{\Delta}+O\left(\hat{\Delta}^{3}\right), \\
& \widehat{E}=\frac{3}{2}+\hat{e} \hat{\Delta}^{2}+O\left(\hat{\Delta}^{4}\right), \\
& \widehat{E}_{\infty}=\frac{3}{2}+\hat{e}_{\infty} \hat{\Delta}^{2}+O\left(\hat{\Delta}^{4}\right), \\
& \hat{j}=\hat{j}_{0} \hat{\Delta}+O\left(\hat{\Delta}^{3}\right), \\
& \hat{g}=\hat{g}_{0} \hat{\Delta}+O\left(\hat{\Delta}^{3}\right), \\
& \hat{\omega}=\hat{\omega}_{0} \hat{\Delta}+O\left(\hat{\Delta}^{3}\right) .
\end{aligned}
$$

Substituting expansions (4.6) into relations (4.4 a $-4.4 \mathrm{e}$ ), (4.5), we obtain step by step that

$$
\begin{aligned}
& \hat{u}^{(0)}-\hat{u}_{\infty}^{(0)}=\hat{j}_{0}, \\
& \hat{e}-\hat{e}_{\infty}=-\frac{3}{2} \bar{R}_{2}+\frac{3}{2} \hat{j}_{0} \frac{\hat{\lambda}}{F^{2}\left(\frac{3}{2}\right)}\left(\hat{u}^{(0)}+\hat{u}_{\infty}^{(0)}\right), \\
& \hat{g}_{0}=\Lambda\left(\frac{3}{2}\right) \hat{j}_{0}, \\
& \hat{e}-\hat{e}_{\infty}=-\frac{3}{4} \bar{R}_{2}+\frac{3}{4} \widehat{A} \hat{\lambda} \frac{\hat{j}_{0}}{F^{2}\left(\frac{3}{2}\right)}\left(\hat{u}^{(0)}+\hat{u}_{\infty}^{(0)}\right), \\
& \varepsilon \frac{\hat{j}_{0}}{F\left(\frac{3}{2}\right)}=\hat{\omega}_{0}, \\
& \hat{e}=\frac{3\left(\hat{u}^{(0)}\right)^{2}}{2\left|c\left(\frac{3}{2}\right)\right| F\left(\frac{3}{2}\right)}, \quad \hat{e}_{\infty}=\frac{3\left(\hat{u}_{\infty}^{(0)}\right)^{2}}{2\left|c\left(\frac{3}{2}\right)\right| F\left(\frac{3}{2}\right)} .
\end{aligned}
$$

Analyzing relations (4.7 b), (4.7 d, $4.7 \mathrm{f})$, we deduce the solvability conditions

$$
\begin{aligned}
& \bar{R}_{2}=\frac{(2-\widehat{A}) \hat{j}_{0} \hat{\lambda}}{F^{2}\left(\frac{3}{2}\right)}\left(\hat{u}^{(0)}+\hat{u}_{\infty}^{(0)}\right), \\
& \widehat{A}=1+\frac{F\left(\frac{3}{2}\right)}{\left|c\left(\frac{3}{2}\right)\right| \hat{\lambda}}, \\
& \hat{u}^{(0)}+\hat{u}_{\infty}^{(0)}=-\tilde{\mu} .
\end{aligned}
$$

Here $\tilde{\mu}$ is an arbitrary constant. 
Consequently, it follows from (4.7 a, 4.8 that

$$
\left.\begin{array}{l}
\hat{u}^{(0)}=\frac{1}{2} \hat{j}_{0}-\frac{1}{2} \hat{\mu}, \\
\hat{u}_{\infty}^{(0)}=-\frac{1}{2} \hat{j}_{0}-\frac{1}{2} \hat{\mu}, \\
\bar{R}_{2}=-\hat{j}_{0} \frac{\left(\left|c\left(\frac{3}{2}\right)\right| \hat{\lambda}-F\left(\frac{3}{2}\right)\right) \tilde{\mu}}{\left|c\left(\frac{3}{2}\right)\right| F^{2}\left(\frac{3}{2}\right)} .
\end{array}\right\}
$$

Using relations (4.9) we can easily describe every possible situation taking place for a stationary planar discontinuity. Subsequent expansion terms could be easily found as well.

5. Conclusions. In this paper we tried to reply to the following question: do there exist piecewise smooth solutions to the 2-D MEP hydrodynamical model of charge transport in semiconductors with smooth parts separated by a surface of strong discontinuity? Using a standard approach, we have obtained the system of jump conditions. Considering the important case of planar discontinuity, we discussed the legitimacy of the introduction of surface charge and surface current in the Rankine-Hugoniot jump conditions. Some interesting effects take place when we take into account the presence of surface charge, surface current, and surface energy flux. In particular, we discover the electron flow choking effect.

6. Acknowledgements. The author is indebted to R. S. Bushmanov and Yu. L. Trakhinin for help in numerical calculations and the preparation of this manuscript. The author is also indebted to Prof. V. Romano for his critical remarks. This work was supported by the Russian Foundation for Basic Research (06-08-00384 and 07-01-00585).

Appendix. The functions $c, F, G, \Lambda$, and $U$.

The expressions for the production terms have been obtained in 2] (for more details the interested reader is referred to [16, 17]).

The production term of the energy balance equation can be written as

$$
\widetilde{C}_{W}=C_{W} \frac{L}{m^{*} C_{0}^{3}}=\sum_{A=1}^{6} \tilde{\mu}_{A} \sqrt{\zeta_{A}} K_{1}\left(\zeta_{A}\right) \Delta_{A}\left(\zeta_{A}\right),
$$

where

$$
\tilde{\mu}_{A}=\frac{\left(m^{*}\right)^{2}\left(\hbar \omega_{n p}\right)_{A}^{\frac{1}{2}} L\left(D_{t} K\right)_{A}^{2}}{\hbar^{2}\left(K_{B} T_{0}\right)^{\frac{3}{2}} \pi^{\frac{3}{2}} \rho_{0}} z_{A}
$$

$\hbar=\frac{h}{2 \pi}, h$ is the Planck constant, $\left(\hbar \omega_{n p}\right)_{A}$ is the optical phonon energy, $\left(D_{t} K\right)_{A}$ is the deformation potential for optical phonons, $\rho_{0}$ is the mass density of the material, $K_{1}(\zeta)$ is the modified Bessel function of second kind of order one,

$$
\Delta_{A}(\zeta)=n_{B(A)} e^{\zeta}-\left(1+n_{B(A)}\right) e^{-\zeta}, \quad n_{B(A)}=\frac{1}{e^{\xi_{A}}-1}, \quad \zeta_{A}=\frac{3 \xi_{A}}{4 E}, \quad \xi_{A}=\frac{\left(\hbar \omega_{n p}\right)_{A}}{K_{B} T_{0}},
$$

and $z_{A}$ is the number of final equivalent valleys in the intervalley scattering. 
Since

$$
\begin{aligned}
\Delta_{A}\left(\zeta_{A}\right) & =n_{B(A)} e^{-\zeta_{A}}\left(e^{2 \zeta_{A}}-e^{2 \tilde{\zeta}_{A}}\right)=-2 n_{B(A)} e^{\zeta_{A}}\left(\int_{0}^{1} e^{2 \lambda\left(\tilde{\zeta}_{A}-\zeta_{A}\right)} d \lambda\right)\left(\tilde{\zeta}_{A}-\zeta_{A}\right) \\
& =-2 n_{B(A)} \zeta_{A} e^{\zeta_{A}}\left(\int_{0}^{1} e^{2 \lambda\left(\tilde{\zeta}_{A}-\zeta_{A}\right)} d \lambda\right) \frac{P}{R},
\end{aligned}
$$

where $\tilde{\zeta}_{A}=\frac{\xi_{A}}{2}\left(E=\widetilde{E}=\frac{3}{2}\right)$, we have the expression for the coefficient $c$ :

$$
\begin{gathered}
R \widetilde{C}_{W}=c P \\
c=-2 \sum_{A=1}^{6} \tilde{\mu}_{A} n_{B(A)} \zeta_{A}^{\frac{3}{2}} e^{\zeta_{A}} K_{1}\left(\zeta_{A}\right)\left(\int_{0}^{1} e^{2 \lambda\left(\tilde{\zeta}_{A}-\zeta_{A}\right)} d \lambda\right) .
\end{gathered}
$$

Concerning $\mathbf{C}_{\mathbf{P}}, \mathbf{C}_{\mathbf{W}}$, we have

$$
\begin{aligned}
& \left(\begin{array}{l}
C_{P}^{(x),(y)} \\
C_{W}^{(x),(y)}
\end{array}\right)=\left(\begin{array}{cc}
\frac{L}{m^{*} C_{0}^{2}} & 0 \\
0 & \frac{L}{m^{*} C_{0}^{4}}
\end{array}\right)\left[\left(\begin{array}{cc}
a_{11}^{(a c)} & a_{12}^{(a c)} \\
a_{21}^{(a c)} & a_{22}^{(a c)}
\end{array}\right)+\left(\begin{array}{cc}
a_{11}^{(n p)} & a_{12}^{(n p)} \\
a_{21}^{(n p)} & a_{22}^{(n p)}
\end{array}\right)\right] \\
& \cdot\left(\begin{array}{cc}
-\frac{21 m^{*}}{W} & \frac{9 m^{*}}{4 W^{2}} \\
\frac{9 m^{*}}{4 W^{5}} & -\frac{27 m^{*}}{20 W^{3}}
\end{array}\right)\left(\begin{array}{cc}
C_{0} & 0 \\
0 & m^{*} C_{0}^{3}
\end{array}\right)\left(\begin{array}{l}
u^{(x),(y)} \\
q^{(x),(y)}
\end{array}\right)=\left(\begin{array}{cc}
c_{11} & c_{12} \\
c_{21} & c_{22}
\end{array}\right)\left(\begin{array}{l}
u^{(x),(y)} \\
q^{(x),(y)}
\end{array}\right) \\
& =\left(\tilde{\nu}_{a c} \widetilde{\mathcal{A}}+\sum_{A=1}^{6} \tilde{\nu}_{n p(A)} \mathcal{B}_{A}\right)\left(\begin{array}{l}
u^{(x),(y)} \\
q^{(x),(y)}
\end{array}\right),
\end{aligned}
$$

where

$$
\begin{array}{rlrl}
a_{11}^{(a c)} & =\frac{32}{3} \frac{\sqrt{2 \pi}}{\hbar^{3}} K_{a c}\left(m^{*}\right)^{\frac{3}{2}}\left(\frac{2}{3} W\right)^{\frac{3}{2}}, & & a_{12}^{(a c)}=2 W a_{11}^{(a c)} \\
a_{21}^{(a c)} & =\frac{a_{12}^{(a c)}}{m^{*}}, & a_{22}^{(a c)}=\frac{8}{3} W a_{21}^{(a c)}, \\
K_{a c} & =\frac{K_{B} T_{0} \Xi_{d}^{2}}{4 \pi^{2} \hbar \rho_{0} v_{s}^{2}}, & &
\end{array}
$$


$\Xi_{d}$ is the deformation potential of acoustic phonons, $v_{s}$ is the sound speed,

$$
\begin{aligned}
& a_{11}^{(n p)}=\sum_{A=1}^{6} \frac{4}{3}\left(\frac{2}{3} W\right)^{-\frac{1}{2}} \frac{\sqrt{2 \pi}\left(m^{*}\right)^{\frac{3}{2}}\left(\hbar \omega_{n p}\right)_{A}^{2}}{\hbar^{3}} z_{A} K_{n p(A)}\left\{n_{B(A)} e^{\zeta_{A}}\left(K_{2}\left(\zeta_{A}\right)-K_{1}\left(\zeta_{A}\right)\right)\right. \\
& \left.+\left(n_{B(A)}+1\right) e^{-\zeta_{A}}\left(K_{2}\left(\zeta_{A}\right)+K_{1}\left(\zeta_{A}\right)\right)\right\} \\
& a_{12}^{(n p)}=\sum_{A=1}^{6} \frac{4}{3}\left(\frac{2}{3} W\right)^{\frac{1}{2}} \frac{\sqrt{2 \pi}\left(m^{*}\right)^{\frac{3}{2}}\left(\hbar \omega_{n p}\right)_{A}^{2}}{\hbar^{3}} z_{A} K_{n p(A)}\left\{n _ { B ( A ) } e ^ { \zeta _ { A } } \left[3 K_{2}\left(\zeta_{A}\right)\right.\right. \\
& \left.\left.+2 \zeta_{A}\left(K_{1}\left(\zeta_{A}\right)-K_{2}\left(\zeta_{A}\right)\right)\right]+\left(n_{B(A)}+1\right) e^{-\zeta_{A}}\left[3 K_{2}\left(\zeta_{A}\right)+2 \zeta_{A}\left(K_{1}\left(\zeta_{A}\right)+K_{2}\left(\zeta_{A}\right)\right)\right]\right\}, \\
& a_{21}^{(n p)}=\frac{a_{12}^{(n p)}}{m^{*}} \\
& a_{22}^{(n p)}=\sum_{A=1}^{6} \frac{4}{3}\left(\frac{2}{3} W\right)^{\frac{3}{2}} \frac{\sqrt{2 \pi}\left(m^{*}\right)^{\frac{1}{2}}\left(\hbar \omega_{n p}\right)_{A}^{2}}{\hbar^{3}} z_{A} K_{n p(A)} \\
& \left\{n_{B(A)} e^{\zeta_{A}}\left[K_{2}\left(\zeta_{A}\right)\left(12-9 \zeta_{A}+4 \zeta_{A}^{2}\right)+K_{1}\left(\zeta_{A}\right)\left(3 \zeta_{A}-4 \zeta_{A}^{2}\right)\right]\right. \\
& \left.+\left(n_{B(A)}+1\right) e^{-\zeta_{A}}\left[K_{2}\left(\zeta_{A}\right)\left(12+9 \zeta_{A}+4 \zeta_{A}^{2}\right)+K_{1}\left(\zeta_{A}\right)\left(3 \zeta_{A}+4 \zeta_{A}^{2}\right)\right]\right\}, \\
& K_{n p(A)}=\frac{\hbar\left(D_{t} K\right)_{A}^{2}}{8 \pi^{2} \rho_{0}\left(\hbar \omega_{n p}\right)_{A}},
\end{aligned}
$$

$K_{2}(\zeta)$ is the modified Bessel function of second kind of order two,

$$
\begin{aligned}
& \tilde{\nu}_{a c}=\left(\frac{2}{3}\right)^{\frac{3}{2}} \frac{8 L \sqrt{2 \pi}\left(m^{*}\right)^{2}}{\hbar^{3}} K_{a c}, \\
& \tilde{\nu}_{n p(A)}=\frac{\sqrt{2 \pi}\left(\hbar \omega_{n p}\right)_{A}^{2} K_{n p(A)} L}{\sqrt{\frac{2}{3}} \hbar^{3} C_{0}^{4}} z_{A}, \\
& \tilde{\mathcal{A}}=\frac{1}{\sqrt{E}} \mathcal{A}, \quad \mathcal{A}=\left(\begin{array}{cc}
-22 E & -\frac{3}{5} \\
-40 E^{2} & -\frac{18}{5} E
\end{array}\right), \quad \mathcal{B}_{A}=\left(\begin{array}{ll}
b_{11} & b_{12} \\
b_{21} & b_{22}
\end{array}\right) .
\end{aligned}
$$


Here

$$
\begin{gathered}
b_{11}=\frac{\Delta_{A}^{\prime}\left(\zeta_{A}\right)\left[4 \zeta_{A} K_{1}\left(\zeta_{A}\right)-22 K_{2}\left(\zeta_{A}\right)\right]+\Delta_{A}\left(\zeta_{A}\right)\left[28 K_{1}\left(\zeta_{A}\right)-4 \zeta_{A} K_{2}\left(\zeta_{A}\right)\right]}{E^{\frac{3}{2}}}, \\
b_{12}=\frac{-\Delta_{A}^{\prime}\left(\zeta_{A}\right)\left[\frac{3}{5} K_{2}\left(\zeta_{A}\right)+\frac{12}{5} \zeta_{A} K_{1}\left(\zeta_{A}\right)\right]+\Delta_{A}\left(\zeta_{A}\right)\left[\frac{12}{5} \zeta_{A} K_{2}\left(\zeta_{A}\right)-3 K_{1}\left(\zeta_{A}\right)\right]}{E^{\frac{5}{2}}}, \\
b_{21}=E^{-\frac{1}{2}}\left\{\Delta_{A}^{\prime}\left(\zeta_{A}\right)\left[\left(\frac{16}{3} \zeta_{A}^{2}-40\right) K_{2}\left(\zeta_{A}\right)-\frac{100}{3} \zeta_{A} K_{1}\left(\zeta_{A}\right)\right]\right. \\
\left.\quad+\Delta_{A}\left(\zeta_{A}\right)\left[\frac{76}{3} \zeta_{A} K_{2}\left(\zeta_{A}\right)-\frac{16}{3} \zeta_{A}^{2} K_{1}\left(\zeta_{A}\right)\right]\right\}, \\
b_{22}=E^{-\frac{3}{2}}\left\{\Delta_{A}^{\prime}\left(\zeta_{A}\right)\left[\frac{8}{5} \zeta_{A} K_{1}\left(\zeta_{A}\right)-\left(\frac{18}{5}+\frac{16}{5} \zeta_{A}^{2}\right) K_{2}\left(\zeta_{A}\right)\right]\right. \\
\left.\quad+\Delta_{A}\left(\zeta_{A}\right) \frac{16}{5} \zeta_{A}\left[K_{2}\left(\zeta_{A}\right)+\zeta_{A} K_{1}\left(\zeta_{A}\right)\right]\right\}
\end{gathered}
$$

Note that $\Delta_{A}\left(\tilde{\zeta}_{A}\right)=0, \quad \Delta_{A}^{\prime}\left(\tilde{\zeta}_{A}\right)=\frac{2 e^{\tilde{\zeta}_{A}}}{e^{2 \tilde{\zeta}_{A}}-1}$.

The constants appearing in the above relations are reported in Tables 1, 2 [16, 17.

In Table 1 all physical parameters are placed:

Table 1

\begin{tabular}{|c|l|c|}
\hline$m_{e}$ & Electron mass in the vacuum & $9.11 \times 10^{-31} \mathrm{~kg}$ \\
\hline$m^{*}$ & Effective mass of electron & $2.9152 \times 10^{-31} \mathrm{~kg}$ \\
\hline$e$ & Electron charge & $1.6 \times 10^{-19} \mathrm{C}$ \\
\hline$\epsilon$ & Dielectric constant & $1.03545 \times 10^{-10} \frac{\mathrm{C}}{\mathrm{V} \cdot \mathrm{m}}$ \\
\hline$N^{+}$ & & $3 \times 10^{23} \frac{1}{\mathrm{~m}^{3}}$ \\
\hline $\mathbb{C}_{0}$ & Sound speed & $1.19170 \times 10^{5} \frac{\mathrm{m}}{\mathrm{S}}$ \\
\hline$T_{0}$ & Lattice temperature & $300 \mathrm{~K}$ \\
\hline$K_{B}$ & Boltzmann constant & $1.38 \times 10^{-23} \frac{\mathrm{J}}{\mathrm{K}}$ \\
\hline$L$ & & $6 \times 10^{-7} \mathrm{~m}$ \\
\hline$\beta$ & $\beta=\frac{e^{2} L^{2} N^{+}}{\epsilon m^{*} C_{0}^{2}}$ & $6.45 \times 10^{3}$ \\
\hline$\varepsilon$ & $\varepsilon=1 / \beta$ & $1.55 \times 10^{-4}$ \\
\hline$h$ & Planck constant & $6.6254 \times 10^{-34} \mathrm{~J} \cdot \mathrm{s}$ \\
\hline$\hbar$ & $\frac{h}{2 \pi}$ & $1.05447 \times 10^{-34} \mathrm{~J} \cdot \mathrm{s}$ \\
\hline$\rho_{0}$ & Mass density & $2.33 \times 10^{3} \frac{\mathrm{kg}}{\mathrm{m}^{3}}$ \\
\hline$\Xi_{d}$ & Deformation potential & $1.44171 \times 10^{-18} \mathrm{~J}$ \\
\hline$v_{s}$ & Sound speed & $9.18 \times 10^{3} \frac{\mathrm{m}}{\mathrm{s}}$ \\
\hline$K_{a c}$ & & $1.05274 \times 10^{-35} \frac{\mathrm{J} \cdot \mathrm{m}^{3}}{\mathrm{~s}}$ \\
\hline$\tilde{\nu}_{a c}$ & & 4.99755 \\
\hline
\end{tabular}

In Table 2 coupling constants and phonon energies for the inelastic scatterings in silicon are reported: 
Table 2

\begin{tabular}{|c|c|c|c|c|c|c|c|c|}
\hline$A$ & $z_{A}$ & $\left(\hbar \omega_{n p}\right)_{A}(\mathrm{~J})$ & $\left(D_{t} K\right)_{A}\left(\frac{\mathrm{J}}{\mathrm{m}}\right)$ & $\xi_{A}$ & $n_{B(A)}$ & $K_{n p(A)}\left(\frac{\mathrm{J} \cdot \mathrm{m}^{3}}{\mathrm{sec}}\right)$ & $\tilde{\mu}_{A}$ & $\tilde{\nu}_{n p(A)}$ \\
\hline 1 & 1 & $1.922 \times 10^{-21}$ & $8.010 \times 10^{-10}$ & 0.464 & 1.692 & $1.913 \times 10^{-37}$ & 0.037 & $5.506 \times 10^{-3}$ \\
2 & 1 & $2.964 \times 10^{-21}$ & $1.282 \times 10^{-9}$ & 0.716 & 0.956 & $3.176 \times 10^{-37}$ & 0.119 & 0.022 \\
3 & 4 & $3.044 \times 10^{-21}$ & $4.806 \times 10^{-10}$ & 0.735 & 0.921 & $4.349 \times 10^{-38}$ & 0.068 & 0.013 \\
4 & 4 & $7.593 \times 10^{-21}$ & $3.204 \times 10^{-9}$ & 1.834 & 0.190 & $7.748 \times 10^{-37}$ & 4.747 & 1.392 \\
5 & 1 & $9.804 \times 10^{-21}$ & $1.762 \times 10^{-8}$ & 2.368 & 0.103 & $1.815 \times 10^{-35}$ & 40.794 & 13.591 \\
6 & 4 & $9.451 \times 10^{-21}$ & $3.204 \times 10^{-9}$ & 2.283 & 0.114 & $6.225 \times 10^{-37}$ & 5.296 & 1.733 \\
\hline
\end{tabular}

The coefficients $\hat{\mu}, \hat{\nu}$ are reported in Table 3. Note that they do not depend on the choice of characteristic values $L$ and $N^{+}$. Moreover, calculating $\hat{\mu}$ and $\hat{\nu}$, we suppose that $\widehat{R}_{\infty}=1$.

Table 3

\begin{tabular}{|l|l|}
\hline$\hat{\mu}$ & 0.1437 \\
\hline$\hat{\nu}$ & 1.0122 \\
\hline
\end{tabular}

In Fig. 2 we plot $c, F, G, \Lambda$, and $U$ versus energy in the case of the parabolic approximation.
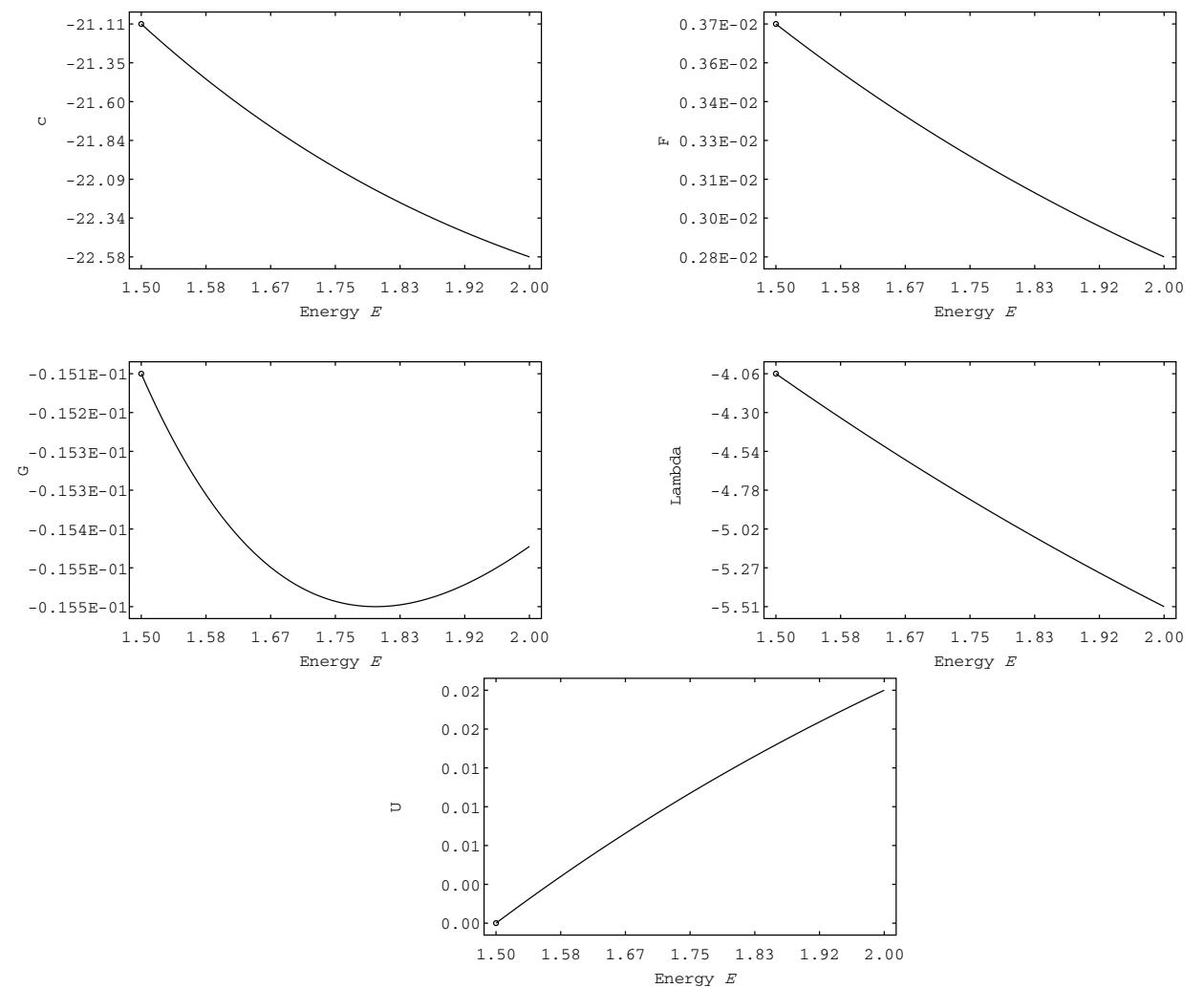

Fig. 2. Functions $c, F, G, \Lambda$, and $U$ versus energy $E$ for the parabolic band approximation 

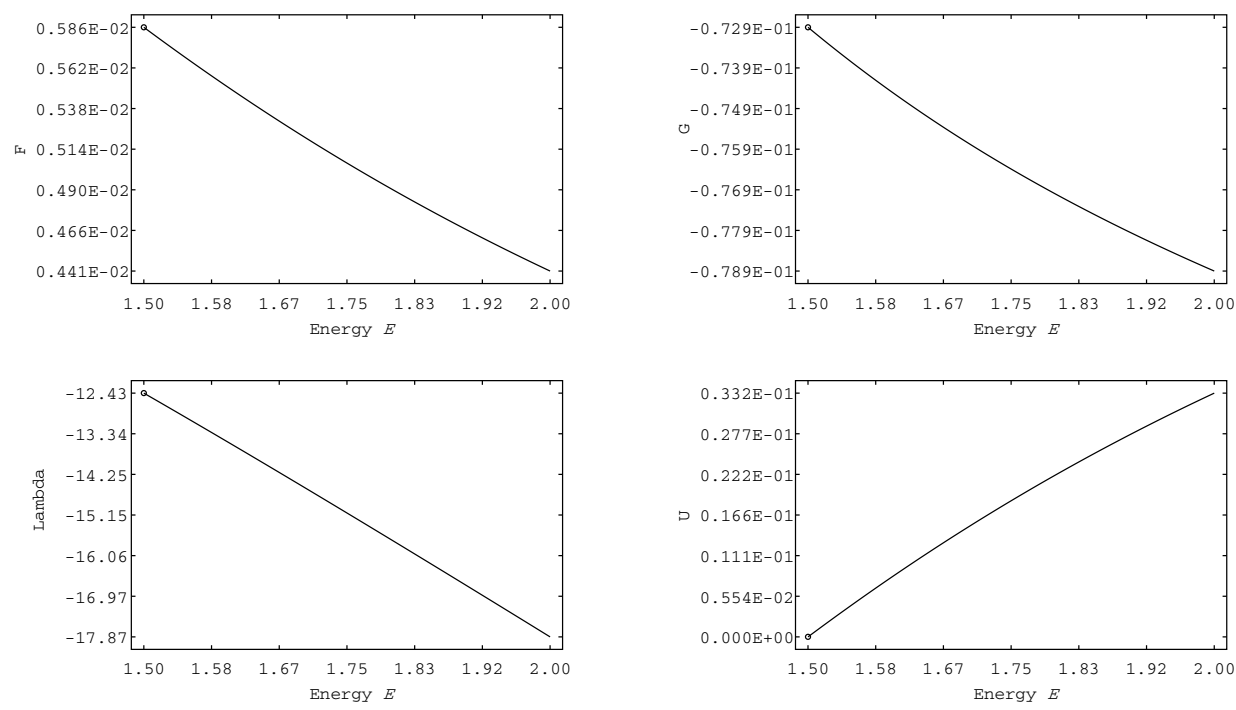

Fig. 3. Functions $F, G, \Lambda$, and $U$ from Section 4

We note that the values of the functions at the equilibrium state (e.g. for $E=\widetilde{E}=\frac{3}{2}$ ) are marked in Fig. 2 by circles.

The graphs of the functions $F, G, \Lambda$, and $U$ from Section 4 are presented in Fig. 3. The value $\widehat{A}=1.896$. Note that it doesn't depend on choice of $L$ and $N^{+}$.

\section{REFERENCES}

[1] A. M. Anile and V. Romano, Non parabolic band transport in semiconductors: closure of the moment equations, Cont. Mech. Thermodyn., 11 (1999), pp. 307-325. MR1723705 (2000j:82044)

[2] V. Romano, Non parabolic band transport in semiconductors: closure of the production terms in the moment equations, Cont. Mech. Thermodyn., 12 (2000), pp. 31-51. MR1753005 (2001g:82117)

[3] S. Selberherr, Analysis and simulation of semiconductor devices, Wien - New York, Springer-Verlag, 1984.

[4] W. Hänsch, The drift-diffusion equation and its applications in MOSFET modeling, Wien, SpringerVerlag, 1991.

[5] P. Markowich, C. A. Ringhofer and C. Schmeiser, Semiconductor equations, Wien, Springer-Verlag, 1990. MR:1063852 (91j:78011)

[6] D. Chen, E. C. Kan, U. Ravaioli, C-W. Shu and R. Dutton, An improved energy-transport model including nonparabolicity and non-maxwellian distribution effects, IEEE on Electron Device Letters, 13 (1992), pp. 26-28.

[7] E. Lyumkis, B. Polsky, A. Shir and P. Visocky, Transient semiconductor device simulation including energy balance equation, COMPEL, 11 (1992), pp. 311-325.

[8] N. B. Abdallah and P. Degond, On a hierarchy of macroscopic models for semiconductors, J. Math. Phys., 37 (1996), pp. 3308-3333. MR.1401227(98b:82091)

[9] A. M. Anile and V. Romano, Hydrodynamical modeling of charge carrier transport in semiconductors, MECCANICA, 35 (2000), pp. 249-296.

[10] A. M. Anile, G. Mascali, V. Romano, Recent developments in hydrodynamical modeling of semiconductors, pp. 1-54 in Mathematical Problems in Semiconductor Physics, Lecture Notes in Mathematics, 1832, Springer, 2003. MR2073497

[11] I. Müller and T. Ruggeri, Rational Extended Thermodynamics, Berlin, Springer-Verlag, 1998. MR.1632151(99h:80001)

[12] D. Jou, J. Casas-Vazquez and G. Lebon, Extended irreversible thermodynamics, Berlin, SpringerVerlag, 1993. MR1271780(95a:80004) 
[13] C. D. Levermore, Moment closure hierarchies for kinetic theories, J. Stat. Phys., 83 (1996), pp. 331-407. MR 1392419 (97e:82041)

[14] A. M. Anile, O. Muscato, V. Romano, Moment equations with maximum entropy closure for carrier transport in semiconductor devices: validation in bulk silicon, VLSI Design, 10 (2000), pp. 335-354.

[15] O. Muscato, V. Romano, Simulation of submicron silicon diode with a non-parabolic hydrodynamical model based on the maximum entropy principle, VLSI Design, 13 (2001), pp. 273-279.

[16] V. Romano, Nonparabolic band hydrodynamical model of silicon semiconductors and simulation of electron devices, Math. Meth. Appl. Sci., 24 (2001), pp. 439-471. MR.1829038 (2002c:82083)

[17] V. Romano, 2D simulation of a silicon MESFET with a non-parabolic hydrodynamical model based on the maximum entropy principle, J. Comp. Phys., 176 (2002), pp. 70-92.

[18] A. M. Blokhin, R. S. Bushmanov, A. S. Rudometova, V. Romano, Linear asymptotic stability of the equilibrium state for the 2-D MEP hydrodynamical model of charge transport in semiconductors, Nonlinear Analysis, 65 (2006), pp. 1018-1038. MR2232491 (2006m:76153)

[19] L. C. Woods, Principles of Magnetoplasma Dynamics, Clarendon Press - Oxford, 1987. MR957923 (89i:76099)

[20] L. V. Ovsyannikov, Lectures on the fundamentals of gas dynamics, Moscow, Nauka, 1981 (Russian). MR665918 (84f:76041)

[21] L. I. Sedov, Continuum mechanics, Vol. 1, River Edge, NJ, World Scientific, 1997.

[22] A. M. Blokhin, R. S. Bushmanov, V. Romano, Asymptotic stability of the equilibrium state for the hydrodynamical model of charge transport in semiconductors based on the maximum entropy principle, Int. J. Eng. Sci., 42(8-9) (2004), pp. 915-934. MR2054625 (2005c:82093)

[23] A. M. Blokhin, R. S. Bushmanov, V. Romano, Nonlinear asymptotic stability of the equilibrium state for the MEP model of charge transport in semiconductors, Nonlinear Analysis, 65 (2006), pp. 2169-2191. MR2266431 (2007i:35225)

[24] A. M. Blokhin, R. S. Bushmanov, V. Romano, Global existence for the system of the macroscopic balance equations of charge transport in semiconductors, J. Math. Anal. Appl. 305 (2005), pp. 72-90. MR 2128112(2005k:35392) 